\title{
Análisis preliminar de los daños ocasionados al maíz por vertebrados plagas en la localidad Pilcos, Colcabamba, Perú
}

Preliminary analysis of damage on maize caused by vertebrates plagas on the Pilcos, Colcabamba, Perú located

${ }^{1}$ Pedro J. García Mendoza ${ }^{a}$ y ${ }^{1}$ Gino P. Prieto Rosales ${ }^{b}$

\section{RESUMEN}

A nivel mundial las aves silvestres representan un grave problema para la producción agrícola, siendo el cultivo de maíz unos de los cereales más apetecidos por diferentes especies granívoras. En este sentido, el principal objetivo de este trabajo fue realizar un análisis preliminar de los daños ocasionados al maíz por vertebrados plagas, utilizando la información generada en un ensayo de evaluación del comportamiento agronómico de 25 variedades de maíz amiláceo provenientes de la región de la sierra peruana. El estudio se llevó a cabo en la localidad Pilcos en el Distrito Colcabamba, Provincia Tayacaja, Perú, en el ciclo del cultivo 2018-2019. El experimento fue establecido bajo un diseño experimental de bloques incompletos Alfa-Látice $5 \times 5$ con tres repeticiones. La unidad experimental estuvo integrada por dos hileras de $4 \mathrm{~m}$ de longitud, separadas a $0,80 \mathrm{~m}$ y $0,20 \mathrm{~m}$ entre plantas dentro de la hilera. El manejo agronómico se hizo conforme a las recomendaciones técnicas establecidas para el cultivo. Durante la etapa del llenado del grano (fases reproductivas R3 y R4) (fase de choclo), se presentó un fuerte ataque de aves (loros), en forma generalizada en todo el ensayo, causando daños severos en todas las entradas del experimento. Se evaluó el daño generado por las aves en las diferentes variedades evaluadas. Los resultados de los análisis estadísticos sugieren algún nivel de preferencia de las aves por las variedades INIA601, Chulpy, Umutu y Choclero-101. En promedio, las aves generaron una reducción en la producción de las variedades de alrededor del 50. Se discuten algunas estrategias para el control de los vertebrados plagas en los cultivos agrícolas.

Palabras clave: maíz, vertebrados plagas, producción agrícola, variedades, aves.

\begin{abstract}
Worldwide, wild birds represent a serious problem for agricultural production, being corn one of the most desirable cereals for different granivorous species. Thus, This study aimed to perform a preliminary analysis of the damage caused to maize by vertebrate pests, using the information generated in the evaluation of the agronomic behavior of 25 varieties of starchy corn from the peruvian Sierra Region. The study was carried out in the Pilcos, Colcabamba District, of the Tayacaja Province, Peru, location, in the 2018-2019 crop season. The trial was established using a incomplete blocks Alpha-Lice 5x5 design with three repetitions.
\end{abstract}


The agronomic management was according to the technical recommendations for the crop. At the grain filling stage (reproductive stages R3 and R4) (choclo phase), there was a strong attack of birds (parrots), in a generalized manner throughout the trial, causing severe damage in all genotype including in the trial. The damage generated by the birds in the different maize varieties was evaluated. The statistical analyzes suggest some level birds preference for the INIA-601, Chulpy, Umutu and Choclero-101 maize varieties. On average, the birds generated a reduction in the maize production around 50\%. Some strategies for the control of vertebrate pests in agricultural crops are discussed.

keyword: corn, vertebrate pests, agricultural production, varieties, birds

${ }^{1}$ Universidad Nacional Autónoma de Tayacaja Daniel Hernandez Morillo, Huancavelica - Perú.

${ }^{\mathrm{a}}$ Ing. Agrónomo; ${ }^{\mathrm{b}}$ Biólogo. 


\section{INTRODUCCIÓN}

Las aves silvestres representan un grave problema para la producción agrícola en el ámbito mundial, particularmente en los cultivos de cereales, tales como maíz, arroz y sorgo (Wards, 1965; Stone, 1972; Casler, et al., 1981). Dolbeer (1990) refiere que una de las especies de aves más abundantes en Norteamérica, Agelaius phoeniceus, vulgarmente conocido como "Mirlo de Alas Rojas", "Tordo Tricolor" o "Sargento Tricolor", genera pérdidas por unas 360,000 t/año, solamente en esa parte del mundo, pero que apenas representa menos del 1\% de la producción de maíz de Norteamérica. No obstante, en los cultivos de soya, trigo, girasol, cebada, quinua y en frutales, las aves también son consideradas una limitante de la productividad, debido a la magnitud de los daños ocasionados en las cosechas de estos cultivos (Bucher y Bedano, 1976; Bruggers y Zaccagnini, 1994; Canavelli et al., 2012).

Para Monge 2007, el conflicto entre las aves y el hombre es quizás tan antiguo como la agricultura. Este investigador señala que entre los daños ocasionados por las aves se encuentran el consumo, contaminación o destrucción de productos agrícolas comestibles, pérdida de productos no comestibles, transmisión de enfermedades, daños en construcciones, interferencia con los niveles de confort en sitios públicos $\mathrm{y}$ daños a la estética de fachadas de edificios y monumentos, además de provocar averías y accidentes de aviación.

En toda Latinoamérica, desde México hasta Argentina, se han reportado daños severos a los cultivos agrícolas causados por las aves (Bucher y Bedano, 1976; Bucher, 1984; Elias y Valencia, 1984). No obstante, a pesar de que en América se han publicado numerosos reportes de aves y mamíferos que provocan daños a los cultivos; Romero-Balderas et al (2006) considera que son escasos los estudios que han evaluado cuantitativamente las pérdidas ocasionadas por estos vertebrados a la agricultura.

Por otro lado, Elias y Valencias (1984) refiere que la apertura de nuevas áreas agrícolas y ganaderas donde existía vegetación nativa ha desencadenado un conflicto entre los humanos y la fauna silvestre, debido a la irrupción de poblaciones silvestres en los cultivos, así como en las ganaderías. En este sentido, Bucher (1992) también señala que en la medida que la frontera agrícola se ha ido expandiendo y ha invadido el bosque tropical, se han intensificado los conflictos entre las aves y la actividad agrícola.

En el Perú, las aves pertenecientes a los géneros Passeriformes (Familias Fringillidae, Ploceidae, Icteridae, Thraupidae, Mimidae y Turdidade), Tinamimorfes, Columbiformes y Psittaciformes, son las más importantes, que han sido señaladas como plagas agrícolas (Aguilar et al., 1977). 
El Distrito Colcabamba representa a uno de los 21 Distritos de la Provincia de Tayacaja, en el Departamento de Huancavelica. Está ubicado en el noreste de la Provincia, entre las coordenadas $12^{\circ}$ 24' 30" S; 74 40' 36" O. La Comunidad Campesina de Pilcos en Colcabamba, cultiva maíz para choclo durante casi todo el año, utilizando riego total o complementario.

En esta localidad, los productores de maíz enfrentan serios problemas con algunas especies de aves silvestres que se alimentan del maíz. En este sentido, el objetivo principal de este estudio fue el de realizar un análisis preliminar de los daños ocasionados al cultivo del maíz por los vertebrados plagas, en la Comunidad Campesina de Pilcos, Colcabamba, Perú, utilizando la información generada en un ensayo de evaluación del comportamiento agronómico de 25 variedades de maíz amiláceo, establecido en un lote de terreno de esta comunidad campesina.

\section{MATERIALES Y MÉTODOS}

\section{Material experimental y ubicación del estudio}

El estudio se llevó a cabo en un lote de terreno perteneciente a la Comunidad Campesina Pilcos en el Distrito de Colcabamba, ubicado entre las coordenadas geográficas - $73^{\circ}-26^{\prime}-21,28^{\prime \prime}$ de longitud Este y $77^{\circ} 44^{\prime} 7.03^{\prime \prime}$ de latitud Norte, aprovechando la información generada en un ensayo de maíz establecido en esta localidad, con el fin de evaluar el comportamiento agronómico de 25 variedades de maíz amiláceo (Tabla 1). Las 25 variedades de maíz fueron establecidas bajo un diseño experimental de bloques incompletos Alfa-Látice 5 x 5 , con tres repeticiones, el día 31 de octubre de 2018.

El desarrollo del cultivo se dio de manera normal, sin mayores problemas, salvo algunos períodos de déficit hídricos en las etapas críticas de prefloración y postfloración, que si bien tuvieron efectos negativos en el desarrollo normal de la planta y de la mazorca, permitió un adecuado llenado de los granos formados en las mazorcas y observar diferencias apreciables en la respuesta de los materiales evaluados.

No obstante, en la época del llenado de grano, en la fase reproductiva entre R3 y R4 (fase de choclo), se presentó un fuerte ataque de aves (loros), en forma generalizada en todo el ensayo, causando daños severos en todas las entradas del ensayo, posiblemente favorecida por el aislamiento de la parcela experimental de otras siembras comerciales de maíz y por la presencia de árboles en los alrededores del ensayo, que representaron el hábitat apropiado para el resguardo de las aves.

De esta manera, se tomó la decisión de utilizar la información para evaluar, de manera preliminar, la magnitud de los daños causados por estas aves al cultivo del maíz. 


\section{Metodología para la evaluación del daño}

Después de efectuada la cosecha, en cada unidad experimental se contó el número de mazorcas totales, el número de mazorcas afectadas por los loros y se escogió una mazorca sana y otra con daños por los loros, para realizar una prueba de medias pareadas, tomando en consideración que ambas mazorcas tuvieran más o menos el mismo nivel de desarrollo. Ambas mazorcas fueron pesadas en una balanza analítica.

En el total de mazorcas cosechadas en cada unidad experimental se formaron dos grupos de mazorcas, siendo el grupo 1 el de las mazorcas sanas y el grupo 2 el de las mazorcas dañadas por las aves. Ambos grupos de mazorcas fueron pesados por separado en una balanza analítica.

Tabla 1. Lista de materiales experimentales y comerciales, lugar de origen, nombre del productor del cultivar y nombre dado a las variedades.

\begin{tabular}{|c|c|c|c|c|}
\hline Entrada & Distrito & Localidad & Productor & $\begin{array}{l}\text { Variedad } \\
\text { recolectada }\end{array}$ \\
\hline 1 & \multirow{4}{*}{$\begin{array}{l}\text { Daniel } \\
\text { Hernández }\end{array}$} & \multirow{2}{*}{ Los Ángeles de Cucharán } & Mauro Aguirre Astill & \\
\hline 2 & & & \multirow{2}{*}{\multicolumn{2}{|c|}{$\begin{array}{l}\text { Alejandro Bobadilla Asti. Blanca } \\
\text { Jaime Salazar Concha Carhuay }\end{array}$}} \\
\hline 3 & & Pata Pampa, Santa María & & \\
\hline 4 & & Rundo & \multicolumn{2}{|c|}{ Adalberto Ega $\quad$ Carhuay } \\
\hline 5 & \multirow{3}{*}{ Quishuar } & Anjara Pata & \multicolumn{2}{|c|}{ Karina Contreras Carhuay } \\
\hline 6 & & Pachas & \multicolumn{2}{|c|}{ María G. Hidalgo Pérez Bolón } \\
\hline 7 & & Yacutoclla & \multicolumn{2}{|c|}{ Richard Vanza Vegas Chulpy } \\
\hline 8 & \multirow{4}{*}{ Pampas } & & \multicolumn{2}{|c|}{ Sergio Pérez Zurca Astilla Blanca } \\
\hline 9 & & Ahuayta Casay & \multirow{3}{*}{\multicolumn{2}{|c|}{$\begin{array}{ll}\text { Gerardo Vilca } & \text { Astilla Blanca } \\
\text { Porfirio Quispe } & \text { Cusqueado } \\
\text { Climaco Culcapuza } & \text { Carhuay }\end{array}$}} \\
\hline 10 & & Gentil Pampas & & \\
\hline 11 & & Manta & & \\
\hline 12 & \multirow{3}{*}{ Colcabamba } & Agua Dulce, Comunidad & Sixto Camasca Asti & \multirow{2}{*}{$\begin{array}{l}\text { Astilla Blanca } \\
\text { Astilla Blanca }\end{array}$} \\
\hline 13 & & Campesina San Julian Alto & Nery Pariona Asti & \\
\hline 14 & & Buenos Aires & \multicolumn{2}{|c|}{ Nicanol Cabana Castro Almidón } \\
\hline 15 & \multirow{3}{*}{$\begin{array}{l}\text { Santiago de } \\
\text { Tucuma }\end{array}$} & Huayrapire & \multicolumn{2}{|c|}{ Moises Pérez Enciso Asti. Blanca } \\
\hline 16 & & Vuelo Pata & \multirow{2}{*}{\multicolumn{2}{|c|}{$\begin{array}{ll}\text { Reymundo Montañez } & \text { Chulpy } \\
\text { Mario Oré Castro } & \text { Chulpy }\end{array}$}} \\
\hline 17 & & Usnupampa & & \\
\hline 18 & \multirow{3}{*}{ Pichos } & & \\
\hline 19 & & Muyupata & \multicolumn{2}{|l|}{ Nora Aliaga Sedana } \\
\hline 20 & & INIA Cajamarca & Alicia Medina $\quad \mathrm{Ch}$ & hoclero-101 \\
\hline 21 & \multirow{4}{*}{ Testigos } & INIA Cajamarca & Alicia Medina & $\begin{array}{l}\text { clero - INIA } \\
\quad 603\end{array}$ \\
\hline 22 & & INIA Cajamarca & Alicia Medina & INIA-601 \\
\hline 23 & & Colección Local Cajamarca & Alicia Medina & Umutu \\
\hline 24 & & Colección Local Cajamarca & Alicia Medina & $\begin{array}{l}\text { aucha Semi } \\
\text { Blanco }\end{array}$ \\
\hline 25 & & Colección Local Cajamarca & Alicia Medina & laucha Rojo \\
\hline
\end{tabular}


Adicionalmente, en cada unidad experimental se determinó el número hileras por mazorcas y número de granos por hileras en ambos grupos de mazorcas (sanas y dañadas por los loros). En el conjunto de mazorcas dañadas también se determinó la proporción de la mazorca dañada por las aves, siendo esta estimación efectuada a través del número de hileras y considerando un número de granos por hileras promedio afectados por las aves.

\section{Análisis de varianza y variables} utilizadas

Con el par de mazorcas sanas y enfermas de cada entrada del ensayo se efectuó una prueba de medias pareadas, aplicando el siguiente modelo matemático (Ecuación 1), conforme a Steel y Torrie (1988).

$$
t=\frac{\left(\overline{\mathrm{y}}_{\mathrm{A}}-\overline{\mathrm{y}}_{\mathrm{B}}\right)-\left(\bar{u}_{\mathrm{A}}-\bar{u}_{\mathrm{B}}\right)}{\sqrt[S]{\left(1 / n_{A}+1 / n_{B}\right)}}[1], \text { donde: }
$$

$\overline{\mathrm{y}}_{\mathrm{A}} \mathrm{y} \overline{\mathrm{y}}_{\mathrm{B}}$ son las medias muestrales, $u_{\mathrm{B}} \mathrm{y} u_{\mathrm{A}}$ representan a las medias poblacionales, $s$ es la desviación típica muestral conjunta, que se distribuyen con, $n_{A}+n_{B}-2$ grados de libertad.

Se realizaron los análisis de varianza respectivo para las variables peso de mazorcas sanas (PMS), peso de mazorcas dañadas (PMD), número de mazorcas dañadas (NMD) y el rendimiento de grano (Rend) a 12\% de humedad, expresado en t/ha, considerando el diseño experimental de Alfa-Látice $5 \times 5$ con tres repeticiones, conforme al siguiente modelo matemático (Ecuación 2):

$Y_{i j k}=\mu+\tau_{i}+R_{j}+\beta_{k(j)}+\varepsilon_{i j k},[2]$, donde:

$Y_{i j k}$ representa la observación correspondiente a la i-ésima variedad, evaluada en laj-ésima repetición y en el $k$ ésimo bloque.

$\mu$ representa al efecto de la media general del ensayo.

$\tau_{i}$ representa al efecto fijo de la $i$-ésima variedad incluida en los ensayos.

$R_{j}$ representa el efecto aleatorio de la $j$ ésima repetición.

$\beta_{k(j)}$ representa al efecto aleatorio del $k$ esimo bloque dentro de la j-ésima repetición.

$\varepsilon_{i j k}$ representa al efecto del error experimental asociado a la observación.

Adicionalmente, la condición de la mazorca se consideró como un factor adicional de variación, lográndose determinar el efecto del daño, dentro de las variedades de maíz amiláceo utilizadas en el estudio, conforme al siguiente modelo matemático (Ecuación $3)$ :

$$
Y_{i j k z=} u_{+} R_{j+} B_{k(j)+} t_{i+m(t) z i++E i j k,[3], \text { donde: }}
$$

$Y_{i j k z}$ representa la observación correspondiente a la i-ésima variedad, evaluada en la j-ésima repetición, en el $k$ ésimo bloquem y la z-ésima mazorca dentro de cada variedad. 
$\mu$ representa al efecto de la media general del ensayo.

$\tau_{i}$ representa al efecto fijo de la i-ésima variedad incluida en los ensayos.

$R_{j}$ representa el efecto aleatorio de la $j$ ésima repetición.

$\beta_{k(j)}$ representa al efecto aleatorio del $k$ esimo bloque dentro de la j-ésima repetición.

$m(t)_{z i}$ representa al efecto aleatorio de la z-esima mazorca dentro de la i-ésima variedad de maíz amiláceo incluida en el ensayo.

$\varepsilon_{i j k z}$ representa al efecto del error experimental asociado a la observación $Y_{i j k z}$

Previa a la realización de los análisis de varianza se realizó la comprobación de los supuestos básicos. El supuesto de normalidad se comprobó mediante la prueba de Wilk y Shapiro, el de homosedasticidad de varianza de los errores a través de la prueba de Barttlet, el de aditividad, a través de la prueba de Tukey y el supuesto de independencia se consideró satisfecho por la aleatorización de los tratamientos en campo y la selección aleatoria de las mazorcas sanas y dañadas, conforme a Steel y Torrie (1988). Las variables PMS, PMD y Rend debieron ser transformadas por el logaritmo con base 10, en tanto que la variable NMD fue transformada por la raíz cuadrada, para cumplir con el supuesto de normalidad, conforme a Steel y Torrie (1988).

\section{Resultados y discusión de resultados}

El análisis de varianza realizado con el grupo de mazorcas sanas y dañadas por los loros reveló que al menos dos de las variedades de maíz evaluadas difirieron en cuanto al peso de las mazorcas sanas (Tabla 2). No obstante, respecto al peso de las mazorcas dañadas por los loros y al número de mazorcas dañadas, las 25 variedades de maíz evaluadas no fueron estadísticamente $(\mathrm{p} \leq 0,05)$ diferentes unas de otras. Esto sugiere que las aves no tuvieron preferencias apreciables por ninguno de los materiales evaluados, causando un efecto similar en todos los genotipos. En lo que respecta al rendimiento de grano, el análisis detectó diferencias altamente significativas $(p<0.01)$ entre los genotipos evaluados, lo cual sugiere que hubo variedades de maíz que se adaptaron mejor a la condición de ambiente del lugar donde fue establecido el ensayo, logrando expresar en mejor forma su potencial productivo.

En el caso de la variable Rend, el análisis además detectó efectos significativos $(\mathrm{p} \leq 0,05)$ para los factores Rep y bloques/Rep (Tabla-2), lo que demuestra la variabilidad existente en el sitio donde se estableció el experimento, por lo que fue apropiado considerar el diseño de bloques incompletos. 
estableció el experimento, por lo que fue apropiado considerar el diseño de bloques incompletos.

Tabla 2. Cuadrados de medios del análisis de varianza efectuado para las variables peso de mazorcas sanas (PMS), peso de mazorcas dañadas (PMD), número de mazorcas dañadas (NMD) y rendimiento de grano (Rend), de 25 variedades de maíz amiláceo evaluadas en lalocalidad Pilcos, Colcabamaba, en el ciclo del cultivo 2018 2019.

\begin{tabular}{cccccc}
\hline \multirow{2}{*}{ Fuente de Variación } & GL & \multicolumn{4}{c}{ Cuadrados Medios } \\
& & PMS & PMD & NMD & Rend \\
\hline Repetición (Rep) & 2 & 0.1483 & 0.0728 & 0.2924 & $0.3906^{*}$ \\
Bloques /(Rep) & 12 & 0.0418 & 0.0415 & 0.6024 & $0.0596^{*}$ \\
Variedades & 24 & $0.0612 *$ & 0.0602 & 0.5990 & $0.0944^{* *}$ \\
Error & 36 & 0.0316 & 0.0455 & 0.3553 & 0.0231 \\
Total & 74 & & & & \\
& CV & 9.31 & 13.39 & 19.39 & 4.54 \\
& Mvar & 91.530 & 45.129 & 9.973 & 2.649 \\
\hline
\end{tabular}

$* \mathrm{y} * *$ indican diferencias estadísticas al 5\% y $1 \%$, respectivamente

Con los análisis realizados para el grupo de mazorcas sanas versus el grupo de mazorcas afectadas por los loros, en la variable condición de mazorcas (sanas o dañadas) se encontró diferencias altamente significativas $(\mathrm{p}<0.01)$ para el efecto tipo de mazorcas (Tabla 3), lo que significa que el peso de las mazorcas sanas fue muy diferente al peso observado en el grupo de mazorcas dañadas por las aves. No sucedió lo mismo con las variables $\mathrm{N}^{\circ}$ Hileras y $\mathrm{N}^{\circ}$ Granos/H, en donde el efecto tipo de mazorca resultó no significativo $(p \leq 0.05)$, lo cual demuestra el nivel de homogeneidad de estas variables dentro de cada material evaluado y por ende el grupo de mazorcas sanas de cada variedad no presentó variaciones importantes en el número de hileras y número de granos por hileras, comparado con el grupo de mazorcas dañadas por las aves, lo que a su vez es un fiel reflejo de que ambas variables son caracteres fijados en cada variedad, poco dependientes del ambiente y además, para el momento del ataque de las aves ya ambas variables se habían definido en cada cultivar evaluado.

Cabe resaltar que todas las variables analizadas presentaron coeficientes de variación aceptables para el tipo de caracteres evaluados, lo cual demuestra la idoneidad en la evaluación de dichas variables. Tampoco se observó diferencias significativas $(\mathrm{p} \leq 0.05)$ entre las variedades en lo que respecta a la condición de la mazorca (Tabla 3), lo que demuestra que todos los materiales fueron igualmente afectados por las aves que consumieron el maíz en la etapa de choclo. Para el número de hileras y número de granos por hileras el análisis si reveló diferencias. 
altamente significativas entre las variedades, lo cual es un indicativo de la variabilidad genética presente entre los cultivares evaluados. Estos resultados eran de esperarse, dada la biodiversidad observada entre las variedades incluidas en el estudio.

La prueba de medias pareadas encontró diferencias altamente significativas $(p<0.01)$ entre la media de las mazorcas sanas y la media de las mazorcas dañadas por las aves (análisis no mostrado), corroborando los resultados del análisis de varianza realizado con ambos grupos de mazorcas (sanas y dañadas por las aves). La media de las mazorcas sanas fue de 91.530 gr, mientras que el grupo de las mazorcas dañadas apenas alcanzó una media de 45.129 gr (Tabla 4), lo cual representa una reducción en la producción de alrededor de $50 \%$.

Las 25 variedades de maíz amiláceo difirieron $(\mathrm{p} \leq 0.05)$ en el peso de las mazorcas sanas, lo que de alguna forma es un reflejo de las variaciones en el potencial de rendimiento dentro del grupo de variedades probadas. El PMS varió de 53.344 gr (Variedad Bolón, entrada 6) a 169.781 gr (Variedad Chulpy, entrada 7), mientras que el peso de las mazorcas dañadas tuvo una amplitud de variación de 22.277 gr (Variedad Astilla Blanca, entrada 9) hasta 79.302 gr (Variedad Chulpy, entrada 17) (Tabla 4).

Tabla 3. Cuadrados medios del análisis de varianza efectuado para las variables condición de la mazorca (CondMz), número de hileras $\left(\mathrm{N}^{\circ}\right.$ Hileras) y número de granos por hileras $\left(\mathrm{N}^{\circ}\right.$ Granos/H) de 25 variedades de maíz amiláceo evaluadas en la localidad Pilcos, Colcabamaba, en el ciclo del cultivo 2018-2019.

\begin{tabular}{lcccc}
\hline \multirow{2}{*}{ Fuente de variación } & DF & \multicolumn{3}{c}{ Cuadrados Medios } \\
\hline Repetición (Rep) & 2 & $0.205^{* *}$ & 3.307 & 28.927 \\
Bloc/(Rep) & 12 & $0.066^{*}$ & $4.184^{*}$ & 14.918 \\
Variedades (Var) & 24 & $0.099^{*}$ & $24.557^{* *}$ & $36.611^{* *}$ \\
Tipos de Mazorcas /(Var) & 25 & $0.167^{* *}$ & 2.160 & 9.707 \\
Error & 86 & 0.035 & 2.068 & 9.447 \\
Total & 149 & & & \\
& $\mathrm{CV}$ & 10.61 & 12.20 & 17.71 \\
& Media & 68.33 & 11.43 & 17.61 \\
\hline
\end{tabular}

$* \mathrm{y} * *$ indican diferencias estadísticas al 5\% y $1 \%$, respectivamente

Aun cuando el análisis de varianza no detectó diferencias estadísticas importantes para el número de mazorcas dañadas por las aves, se observó una amplitud de variación desde alrededor de 4 mazorcas (Variedad Carhuay, entrada 3), hasta alrededor de 19 mazorcas afectadas (Variedad INIA-601, entrada 22), lo cual se ubica fuera del intervalo de confianza para la media de esta variable, indicando que ambos valores extremos serían 
estadísticamente $(\mathrm{p} \leq 0.05)$ diferentes. En este sentido, las entradas 5 (Carhuay), 9 (Astilla Blanca), 8 (Astilla Blanca), 1 (Astilla Amarilla) y 4 (Carhuay) fueron las que presentaron menor número de mazorcas dañadas, mientras que las entradas 22 (INIA-601), 7 (Chulpy), 23 (Umutu) y 20 (Choclero-101) fueron las variedades que resultaron más afectadas por las aves (Tabla 4), siendo ambos grupos estadísticamente $(\mathrm{p} \leq 0.05)$ diferentes entre sí y con el resto de las variedades. Estos resultaros sugieren algún nivel de preferencia de las aves por las variedades INIA-601, Chulpy, Umutu y Choclero-101.

En lo que respecta al rendimiento de grano, la variedad INIA-601 resultó con el mayor potencial productivo, con 8.114 t/ha, siendo este rendimiento estadísticamente muy superior $(p<0.01)$ al resto de las variedades probadas (Tabla 4). Le siguieron, en orden de importancia, las entradas 17 (Chulpy), 7 (Chulpy), 10 (Cusqueado), 16 (Chulpy), 24 (Chaucha Semi Blanco) y 21 (Choclero INIA-603), cuyos rendimientos se ubicaron entre 3 y 4 t/ha, no habiendo diferencias estadísticas $(p \leq 0.05)$ entre ellas.

Tabla 4. Valores medios del peso de las mazorcas sanas (PMS), peso de mazorcas dañadas (PMD) y el rendimiento de grano de 25 variedades de maíz amiláceo evaluadas en la localidad Pilcos, Colcabamba, en el ciclo del cultivo 2018- 2019.

\begin{tabular}{|c|c|c|c|c|c|}
\hline Entrada & $\begin{array}{l}\text { Variedad } \\
\text { recolectada }\end{array}$ & $\begin{array}{l}\text { PMS } \\
\text { (gr) }\end{array}$ & $\begin{array}{c}\text { PMD } \\
\text { (gr) }\end{array}$ & $\begin{array}{l}\text { NMD } \\
\left(\mathbf{N}^{\circ}\right)\end{array}$ & $\begin{array}{l}\text { Rendimiento } \\
\text { (t/ha) }\end{array}$ \\
\hline 1 & Astilla Amarilla & 55.411 & 37.296 & 6.200 & 1.529 \\
\hline 2 & Astilla Blanca & 78.127 & 24.866 & 9.533 & 1.932 \\
\hline 3 & Carhuay & 69.119 & 26.869 & 8.467 & 1.904 \\
\hline 4 & Carhuay & 56.785 & 35.453 & 7.767 & 1.912 \\
\hline 5 & Carhuay & 96.889 & 33.298 & 4.067 & 1.524 \\
\hline 6 & Bolón & 53.344 & 47.417 & 8.233 & 1.468 \\
\hline 7 & Chulpy & 169.781 & 65.255 & 14.567 & 4.126 \\
\hline 8 & Astilla Blanca & 56.653 & 41.467 & 6.033 & 1.784 \\
\hline 9 & Astilla Blanca & 83.776 & 22.277 & 5.533 & 1.314 \\
\hline 10 & Cusqueado & 155.112 & 49.445 & 11.333 & 3.418 \\
\hline 11 & Carhuay & 67.745 & 37.053 & 11.000 & 1.882 \\
\hline 12 & Astilla Blanca & 128.351 & 51.634 & 10.667 & 1.974 \\
\hline 13 & Astilla Blanca & 62.942 & 44.033 & 8.667 & 2.240 \\
\hline 14 & Almidón & 96.507 & 70.148 & 8.533 & 2.215 \\
\hline 15 & Astilla Blanca & 71.535 & 42.917 & 9.700 & 2.497 \\
\hline 16 & Chulpy & 141.887 & 68.636 & 10.733 & 3.403 \\
\hline 17 & Chulpy & 105.215 & 79.302 & 10.467 & 4.187 \\
\hline 18 & Pistiada & 55.263 & 23.946 & 9.367 & 2.058 \\
\hline 19 & Carhuay & 93.552 & 43.861 & 11.600 & 2.459 \\
\hline 20 & Choclero-101 & 59.402 & 39.418 & 12.067 & 2.697 \\
\hline 21 & $\begin{array}{l}\text { Choclero-INIA- } \\
603\end{array}$ & 99.665 & 30.812 & 11.833 & 3.108 \\
\hline
\end{tabular}


maduración del grano y en los períodos previos a la cosecha. En este sentido, González (1998) encontró que los productores agrícolas que manifestaron tener pérdidas superiores al $30 \%$ reconocieron que no podían vigilar a sus cultivos diariamente, debido a diferentes razones. González (1998) reportó pérdidas en el cultivo del maíz que fueron desde alrededor de 14\% hasta cerca de $70 \%$ de la producción en el noreste de la Amazonia peruana, departamento de Loreto.

Tabla 5. Promedio del peso de mazorcas (PesoMz), del número de hileras en la mazorca (NH) y número de granos por hileras (NGH), de 25 variedades de maíz amiláceo evaluadas en la localidad Pilcos, Colcabamba,

\begin{tabular}{|c|c|c|c|c|}
\hline Entrada & Variedad & $\begin{array}{c}\text { PesoMz } \\
\text { (gr) }\end{array}$ & $\begin{array}{l}\mathrm{NH} \\
\left(\mathrm{N}^{\circ}\right)\end{array}$ & $\begin{array}{c}\text { NGH } \\
\left(\mathrm{N}^{\circ}\right)\end{array}$ \\
\hline 1 & Astilla Amarilla & 46.35 & 10.83 & 16.77 \\
\hline 2 & Astilla Blanca & 51.50 & 10.10 & 17.72 \\
\hline 3 & Carhuay & 47.99 & 9.70 & 15.70 \\
\hline 4 & Carhuay & 46.12 & 11.97 & 15.18 \\
\hline 5 & Carhuay & 65.09 & 10.90 & 16.38 \\
\hline 6 & Bolón & 50.38 & 10.87 & 16.63 \\
\hline 7 & Chulpy & 117.52 & 17.80 & 22.25 \\
\hline 8 & Astilla Blanca & 49.06 & 11.53 & 16.88 \\
\hline 9 & Astilla Blanca & 53.03 & 12.00 & 16.33 \\
\hline 10 & Cusqueado & 102.28 & 8.10 & 20.17 \\
\hline 11 & Carhuay & 52.40 & 12.57 & 16.52 \\
\hline 12 & Astilla Blanca & 89.99 & 12.33 & 16.03 \\
\hline 13 & Astilla Blanca & 53.49 & 12.90 & 13.58 \\
\hline 14 & Almidón & 83.33 & 8.97 & 19.25 \\
\hline 15 & Astilla Blanca & 57.23 & 9.27 & 18.78 \\
\hline 16 & Chulpy & 105.26 & 14.90 & 20.15 \\
\hline 17 & Chulpy & 92.26 & 14.70 & 21.32 \\
\hline 18 & Pistiada & 39.60 & 10.23 & 14.65 \\
\hline 19 & Carhuay & 68.71 & 10.80 & 15.60 \\
\hline 20 & Choclero-101 & 49.41 & 9.53 & 16.37 \\
\hline 21 & Choclero-INIA-603 & 65.24 & 8.43 & 18.43 \\
\hline 22 & INIA-601 & 100.65 & 10.90 & 25.35 \\
\hline 23 & Umutu & 62.25 & 11.87 & 18.23 \\
\hline 24 & Chaucha Semi Blanco & 83.49 & 10.67 & 15.67 \\
\hline \multirow[t]{6}{*}{25} & Chaucha Rojo & 75.60 & 13.80 & 16.38 \\
\hline & Media & 68.33 & 11.43 & 17.61 \\
\hline & Mínimo & 39.604 & 8.100 & 13.583 \\
\hline & Máximo & 117.518 & 17.800 & 25.350 \\
\hline & LSD & 34.673 & 1.598 & 3.5757 \\
\hline & ICM (0.95) & $52.74-83.92$ & $10.71-12.15$ & $16.01-19.22$ \\
\hline
\end{tabular}

LSD e ICM significan mínima diferencia significativa e intervalo de confianza de la media, respectivamente. 


\begin{tabular}{rccccc}
22 & INIA-601 & 126.090 & 75.219 & 19.267 & 8.114 \\
23 & Umutu & 85.944 & 38.561 & 13.067 & 2.492 \\
24 & Chaucha Semi & 112.742 & 54.231 & 11.567 & 3.146 \\
25 & Blanco & 106.407 & 44.800 & 9.067 & 2.833 \\
\hline Chaucha Rojo & Media & $\mathbf{9 1 . 5 3 0}$ & $\mathbf{4 5 . 1 2 9}$ & $\mathbf{9 . 9 7 3}$ & $\mathbf{2 . 6 4 9}$ \\
Mínimo & $\mathbf{5 3 . 3 4 4}$ & $\mathbf{2 2 . 2 7 7}$ & $\mathbf{4 . 0 6 7}$ & $\mathbf{1 . 3 1 4}$ \\
Máximo & $\mathbf{1 6 9 . 7 8 1}$ & $\mathbf{7 9 . 3 0 2}$ & $\mathbf{1 9 . 2 6 7}$ & $\mathbf{8 . 1 1 4}$ \\
& LSD & $\mathbf{6 1 . 6 5 4}$ & $\mathbf{3 6 . 8 5 9}$ & $\mathbf{5 . 9 5 2}$ & $\mathbf{1 . 6 6 8 1}$ \\
& ICM (0.95) & $\mathbf{7 2 . 3 5}-\mathbf{1 1 0 . 7 1}$ & $\mathbf{3 3 . 7 9}-\mathbf{5 6 . 4 7}$ & $\mathbf{8 . 1 4}-\mathbf{1 1 . 8 0}$ & $\mathbf{2 . 1 4}-\mathbf{3 . 1 6}$ \\
\hline
\end{tabular}

LSD e ICM significan mínima diferencia significativa e intervalo de confianza de la media, respectivamente.

Del mismo modo, el peso medio de mazorcas (sanas y dañadas) presentó una amplitud de variación de 39.604 gr a $117.518 \mathrm{gr}$, siendo las entradas 7 (Chulpy), 16 (Chulpy), 10 (Cusqueado) y 22 (INIA-601) las que presentaron los mayores pesos cuando se promedió el peso de las mazorcas sanas y el de las mazorcas dañadas, que se ubicaron alrededor de los 100 gr (Tabla 5).

Por otro lado, el número de hileras en la mazorca en las variedades estudiadas varió de alrededor de 8 hasta 18 hileras por mazorca, siendo las variedades Chulpy (entradas 7, 16 y 17) las que presentaron mayor número de hileras por mazorca, superando estadísticamente $(p \leq 0.05)$ al resto de las variedades en este carácter. Por su parte, el número de granos por mazorcas varió de 13.583 a 25.350 granos por mazorca, en donde la variedad INIA-601 resultó la variedad con las mazorcas con el mayor número de granos promedios en la hilera, superando estadísticamente $(\mathrm{p} \leq 0.05)$ a la mayoría de las variedades restantes probadas (Tabla $5)$.

121 Rev. Tayacaja 2(1): $111-126,2019$ ISSN:2617-9156
En promedio, las aves generaron una reducción en la producción de las variedades de alrededor del $50 \%$, lo cual concuerda con algunas estimaciones realizadas en trabajos previos en el Perú y en otras latitudes del mundo. Por ejemplo Aguilar (1977) señala que las principales especies de aves que causan daños a los cultivos de cereales en el Perú pueden llegar a generar pérdidas superiores al $30 \%$ de la producción en algunas zonas del Perú. Sin embargo, González (1998) menciona que en las márgenes de los grandes ríos de la región amazónica se considera que las aves pueden llegar a causar daños económicos considerables a los pequeños productores y colonos de la región, principalmente a los cultivos de maíz y arroz, pero hasta la fecha no se había realizado ningún estudio específico, que permitiera determinar el nivel real de las pérdidas.

La única manera de que muchas de estas aves no causen pérdidas significativas en los campos de producción es estableciendo un plan de vigilancia permanente durante las etapas de 
Así mismo, Tala et al (2005) evaluaron el daño potencial que podría causar la Cotorra Argentina (Myiopsiptta monachus) sobre la producción agrícola en Chile y concluyen que si bien para el momento del estudio la mayoría de los sitios reproductivos de la especie se encontraban en áreas urbanas, es perfectamente factible que colonicen áreas rurales, donde podrían causar muchos daños a especies de árboles frutales y al maíz.

Esta misma especie fue estudiada en México, en donde se aplicaron encuestas a varias comunidades rurales, encontrándose que este tipo de aves produce daños principalmente en el cultivo de maíz, que, en forma global, oscilaron entre $8 \%$ y $50 \%$. Otros trabajos también han reportado pérdidas agrícolas importantes en el cultivo del maíz y otros cereales, ocasionados por aves y otros vertebrados plagas (Casler et al., 1981; Villar-González, 2000; Robles et al., 2003; Romero-Balderas, et al., 2006; Pérez y Pacheco, 2014; Castillo-López et al., 2017).

Se han propuesto diferentes alternativas para el manejo de los problemas de vertebrados plagas en Latinoamérica. Dentro de estas alternativas se ha sugerido el control letal, práctica que a cada día recibe mayores cuestionamientos (Canavelli et al., 2012). Por esta razón, actualmente las alternativas de manejo que mayor auge están tomando en el ámbito mundial incluyen principios de manejo integral, a escala regional y manejo adaptativo (Bomford y Sinclair, 2002; Parkes et al. 2006). Sin embargo, en lo que respecta al manejo integrado, no existen reportes concretos en los que se hayan aplicado y evaluado el manejo según estos principios (Canavelli et al., 2012). Según Canavelli et al. (2012), entre las alternativas de manejo integrado se proponen algunas medidas agronómicas, dentro de las cuales destacan: 1. Cambiar o coordinar fechas de siembra, lo cual no siempre es factible en todas las regiones; 2. Concentrar los cultivos de una región en áreas extensas o aumentar el tamaño de los lotes individuales; 3. Utilizar cultivares menos susceptibles, lo cual no siempre es posible; 4. Disminuir la disponibilidad de granos en los rastrojos, durante el traslado del producto de las unidades de producción a los centros de recepción, a causa de los cultivos abandonados en pie o los destinados para la alimentación animal; 5. Sustituir cultivos atractivos por pasturas $\mathrm{u}$ otros menos susceptibles; 6. Siembra de cultivos atractivos al vertebrado plaga en las cercanías de los nidos o sitios de concentración, de menor valor que el cultivo principal (cultivo trampa o de distracción).

Una de las medidas más utilizadas y que ha surtido buen efecto en la mayoría de los países y regiones es la protección del cultivo mediante el uso de diferentes métodos, cuyo propósito es disminuir la atracción de los cultivos por las aves granívoras. Dentro de las medidas se pueden indicar métodos físicos, 
generando ruidos extremos con cañones de explosión, pirotecnia, disparos de escopeta, sonidos electrónicos, gritos de aves rapaces; métodos visuales, utilizando figuras disuasivas, tales como globos, siluetas de aves rapaces, cintas reflectoras, excluyendo las aves del cultivo mediante el uso de redes para cubrir el cultivo; métodos químicos, a través del uso de sustancias repelentes o métodos biológicos, mediante el uso de aves rapaces entrenadas o a través del uso de personas que recorren los lotes espantando las aves (Canavelli, 2012),

Sin embargo, vale acarar que todos estos métodos presentan algunas limitaciones. Por ejemplo, la mayoría tienen su aplicación en pequeñas áreas o para cultivos intensivos de alto valor. En el caso de los repelentes, no funcionan bien para todas las especies de aves, siendo algunos muy específicos para determinadas especies.

Otras alternativas propuestas incluyen la modificación del hábitat, que procura hacer el medio menos propicio para las poblaciones de vertebrados plagas. Esta alternativa incluye la disminución de la disponibilidad de recursos alimenticios, discutida previamente y la realización de podas selectivas en los árboles, haciéndolos menos atractivos para las actividades de nidificación (Volpe y Aramburú, 2011).

No obstante, cualquier medida de control a aplicar requiere conocer cuánto se pierde en realidad, para poder evaluar de manera objetiva el beneficio potencial obtenido con la aplicación de dicha medida.

\section{CONCLUSIONES}

A partir de los resultados del presente estudio se pueden generar las siguientes conclusiones generales:

La variedad INIA-601 resultó con el mayor potencial productivo, cuyo rendimiento resultó estadísticamente superior al resto de las variedades probadas, siendo además la variedad con el mayor número de granos promedios por mazorcas.

Las variedades Carhuay (entradas 4 y 5), Astilla Blanca(entradas 8 y 9)y Astilla Amarilla (entrada 1) presentaron menor número de mazorcas dañadas, comparado con las variedades INIA-601 (entrada 22), Chulpy(entrada 7), Umutu (entrada 23) y Choclero-101 (entrada 20), quienes resultaron las más afectadas por las aves, siendo ambos grupos estadísticamente diferentes entre sí y con el resto de las variedades.

En promedio, las aves generaron una reducción en la producción de las variedades de alrededor del $50 \%$, lo que concuerda con algunas estimaciones realizadas en trabajos previos.

Los resultaros sugieren algún nivel de preferencia de las aves por las variedades INIA-601, Chulpy, Umutu y Choclero101. Se han propuesto diferentes alternativas para el manejo de los problemas de vertebrados plagas en Latinoamérica, pero todas ellas presentan serias limitaciones para su aplicación 
en las diferentes regiones. De cualquier forma, toda medida de control a ser aplicada requiere conocer cuánto se pierde en realidad, para poder evaluar de manera objetiva el beneficio potencial obtenido con la aplicación de dicha medida.

\section{REFERENCIAS BIBLIOGRÁFICAS}

Aguilar, P. G., Beingolea, O. D., Brack, A. J. y Ceballos I. 1977. Vertebrados importantes en la agricultura peruana. Revista Peruana de Entomología. 20:25-31.

Bruggers, R. L. y Zaccagnini, M. E. 1994. Vertebrate pest problem related to agriculture production and applied research in Argentina. Vida Silvestre Neotropical, 3. 71-83.

Bucher, E. H. 1984. Las aves como plagas en la Argentina. Publicación $\mathrm{N}^{\circ}$ 9, Centro de Zoología Aplicada. Universidad Nacional de Córdova, Córdova, Argentina.

Bucher, E. H. y Bedano, P- E. 1976. Bird damage problem in Argentina. International Studies on Sparrows. 9:3-16.

Canavelli, S. B., Aramburú, R. M. y Zaccagnini, M. E. 2012. Aspectos a considerar para disminuir los conflictos entre las cotorras (Myiopsitta monachus) y la producción agrícola. Hornero, número especial. Ecología, conservación y manejo de loros en Argentina, 27(1): 89-101.
Casler, C. L, Rivero, A. R. y Lira, J. R. 1981. Los patos Dendrogyna como causantes de daños en el cultivo de arroz en Venezuela (Aves Anatidae). Memoria de la Sociedad de Ciencias Naturales "La Salle". 105 - 115. D is p o n ible e n : http://www.fundacionlasalle.org.ve /userfiles/men_1981_41_115_105115.pdf

Castillo-López, I. F., RodríguezAfricano, P. E., Montes-Pérez, R. C. y González-Valderrama, D. M. 2017. Fauna silvestre que afecta los cultivos en Boyacá y control del daño a cultivos de maíz. Rev. Cien. Agri., 14(1): 75-84.

Elias, D. J. Y Valencia, D. 1984. La agricultura Latinoamericana y los vertebrados plagas. Interciencia 9: $223-229$.

González, J. A. 1998. Análisis preliminar de los daños causados por las aves silvestres a la agricultura en la Amazonia Oriental del Perú. Agencia Española de Cooperación Internacional, Proyecto Araucaria Colca, Miguel Dasso, $117-2^{\circ}$, San Isidro, Lima, Perú. 370 - 380. D i s p o ible e n : http://www.comfauna.org/wpcontent/uploads/2012/PDFsManejofaunasilvestre/Cartagena2001/8_Especies-introducidase s p e c i e s - p 1 a g a / 370 380 jgonzalez AnalisisPreliminar DanosCausadosAvesSilvestres.pdf. Dolbeer, R. A. 1990. Ornithology and 
integrated pest management: Red-winged Blackbirds Agelaius phoeniceus and corn. Ibis, 132(2): 309-322.

Romero-Balderas, K. G., Naranjo, E. J., Morales, H. H. y Nigh, R. B. 2006. Daños ocasionados por vertebrados silvestres al cultivo de maíz en la Selva Lacandona, Chiapas, México. Interciencia, 31(4): 276- 283.

Steel, R. y Torrie, J. 1998. Biostadítica: Principios y Procedimientos. McGraw-Hill/Interamericana de México, S. A. de C. V. 2da. Ed. (Primera en español). P 622. D i s p o n b e e n : http://clea.edu.mx/biblioteca/Steel $\% 20 \mathrm{R}$ o b e r t \% $20 \mathrm{G} \% 20$ $\% 20$ Bioestadistica $\% 20$ Principios $\%$ 20Y\%20Procedimientos\%202ed.pd f.

Tala, C., Guzmán, P. y González, S. 2005. Cotorra Argentina (Myipsiptta mobachus) convidado de piedra en nuestras ciudades y un invasor potencial, aunque real, de sectores agrícolas. Servicio Agrícola y Ganadero- División de los Recursos Naturales Renovables. Boletín DIPROREN. 1 -7pp. Disponible en: http://www.redalyc.org/pdf/756/756 49935007.pdf.

Villar-González. 2000. Principales vertebrados plagas en México: situación actual y alternativas para su manejo. Revista Chapingo Serie Ciencias Forestales y del Ambiente, $6(1): 41-54$.
Volpe, N y Aramburú, R. 2011. Preferencias de nidificación de la Cotorra Argentina (Myiopsitta monachus) en un área urbana de Argentina. Ornitología Neotropical, 22: $111-119$.

\section{CORRESPONDENCIA}

Blgo. Pedro García Mendoza

pedro.garcia@unat.edu.pe 